\title{
Novel Photosensitizer and Methoxy Styryl Pyridines for Photoradical Initiator System
}

\author{
Xuesong Jiang, Jie Yin, Yasuharu Murakami*, and Makoto Kaji* \\ Shanghai Jiao Tong University, School of Chemistry and Chemical Technology \\ 800 Dongchuan Road, Shanghai, 200240 China \\ *Hitachi Chemical Co., Ltd., Research and Development Center, \\ 13-1 Higashi-cho 4-chome, Hitachi-shi, Ibaraki-ken, 317-8555 Japan
}

\begin{abstract}
Distyryl pyridine (DSP) photosensitizer for HABI photoinitiator systems was newly designed and eleven methoxy DSP(distyrylpyridines)s were synthesized and evaluated. The results showed that 2, 4-dimethoxy DSP (24DMOP-DSP) was the most efficient among studied methoxy DSPs. Compared with coumarin 1 as reference, the photosensitivity for 24DMOP-DSP as photosensitizer was increased by $150 \%$. Potential of DSP as photosensitizer was supported by the mechanism study, such like photobleaching property of 24DMOP-DSP, derived from configuration change of $\boldsymbol{E}$ to $\boldsymbol{Z}$, and efficient fluorescence quenching of excited singlet DSP by BCIM.
\end{abstract}

Keyword: photoresist, photoradical polymerization, distyryl pyridine

\section{Introduction}

Photopolymerization science and technology have assumed in recent years an increasing relevance in many applications, such as curing of coatings on various materials, adhesives, printing inks, and photoresists [1-3]. This technology is based on the use of photoinitiator systems suited to absorb a light radiation of the appropriate wavelength and to produce primary radical species able to convert a multifunctional monomer into a crosslinked network. Therefore, photoinitiator system plays a very important role in the photopolymerization science and technology. Among the most photoinitiator systems, hexaarylbiimidazole (HABI or Lophine dimer, L2) have been widely used as free radical initiators in photopolymer imaging, such as proof paper, dry film resist etc[4-8]. The application of HABI is based on well-established properties of its photolytic dissociation. Under UV irradiation, the homolysis of the excited singlet HABI produces long-lived 2,4,5-triarylimidazolyl radicals (lophyl radicals, $\mathrm{L}^{*}$ ) because of the low $\mathrm{C}-\mathrm{N}$ bond energy. The triarylimidazolyl radicals can abstract hydrogen atoms from hydrogen donor (HD) to generate active radicals, and consequently initiate photopolymerizations of acrylate monomers efficiently according to Scheme 1 . Alternate path via electron transfer from hydrogen donor to the triarylimidazolyl radicals may occur depending on each system`s electronic character.

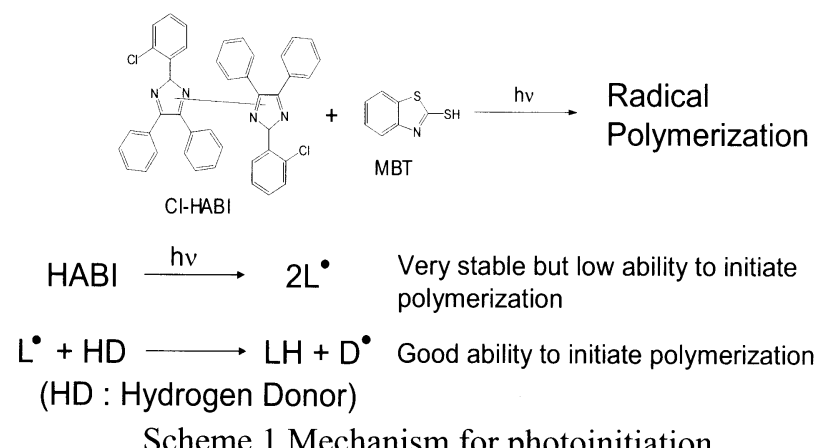

The most widely used HABI derivatives such as BCIM, however, do not possess the satisfied UV absorption property, as their maximum absorption peaks are below $300 \mathrm{~nm}$. Moreover, their extinction coefficients at $365 \mathrm{~nm}$, irradiation 
wavelength most widely utilized for photocuring, are very low. Therefore, it is necessary to add photosensitizer (S) into HABI photoinitiator systems, which can absorb UV light around 365 $\mathrm{nm}$ efficiently and transfer the energy to HABI. Actually, almost all HABI photoinitiator systems in industrial formulation comprise of photosensitizers such as Micheler's ketone (MK), Eosin Y, and Rose Bengal [4]. These photosensitzers can enhance the performance of HABI photointiator systems. Thus, development of efficient photosensitizer is very important to HABI photoinitiator systems in industrial photopolymers, such as dryfilm photoresist, because of requirements from industry such as high sensitivity and high resolution. The efficient photosensitizer should possess two important characters: the first is that phosensitizer can absorb UV light very efficiently and then be excited to the excited state; the other is that the excited state of photosensitizer can transfer its energy to HABI efficiently to produce lophine radicals. For example, as to efficient singlet state's photosensisitzer, compound should possess high fluorescence quantum yield, and its fluorescence can be quenched by HABI efficiently.

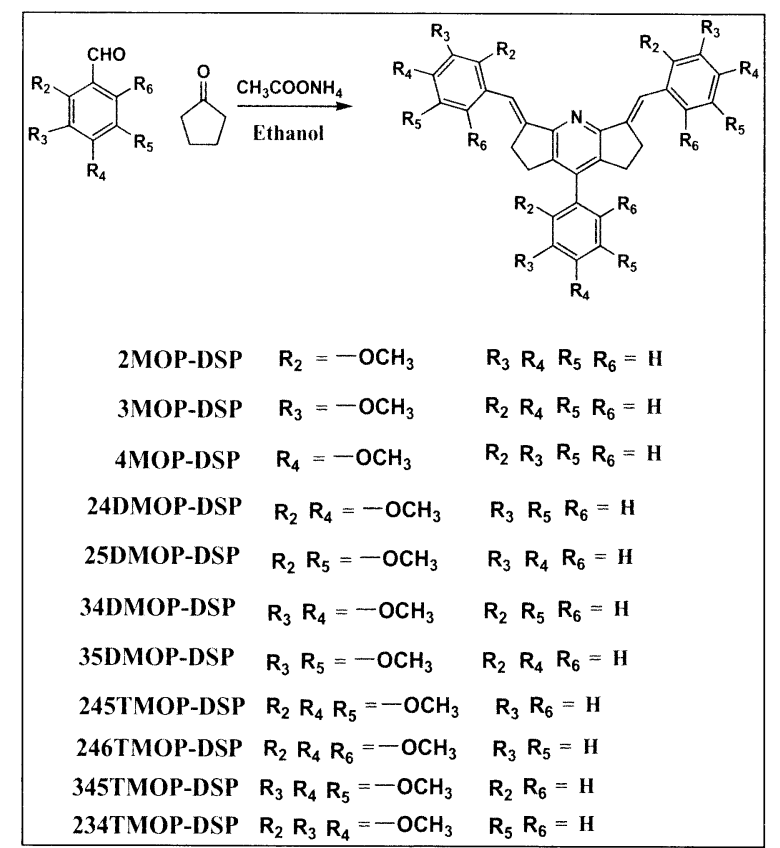

Scheme 2 Structure and synthesis of methoxy DSPs.

Distyrylpyridine derivatives (DSPs) [9] are a new class of fluorescent molecules which are pyridine derivatives with structurally fixed styryl moieties, and can be synthesized through one-step according to Scheme 2. Structural rigidity of DSPs allows us to expect for them high fluorescence quantum yields. In this context, therefore, we studied methoxy-DSPs as photosensitizer for HABI photoinitiator systems in electronic related photopolymers. This report described absorption properties, photosensitivity evaluation, photobleaching character and fluorescence decay analyses of synthesized eleven methoxy DSPs.

Though distyryl pyridines should be called as 3 , 5 -dibenzylidendicyclopentano $[b, e]$ pyridines, in this report these compounds are called as DSPs for short, such like, 24DMOP-DSP for 3, 5-bis (2, 4-dimethoxybenzylidenedicyclopentano $\left[\begin{array}{ll}b, & e\end{array}\right)$, 4-2,4-dimethoxyphenyl pyridine.

\section{Experimental}

2.1 Syntheses of methoxy DSPs

All methoxy DSPs were synthesized through one-step according to Scheme 2, and confirmed by NMR and FT-IR. Using 24DMOP-DSP as example, the detailed process of synthesis is as following: A mixture of 2,4-dimethoxybenzaldehyde $(0.1 \mathrm{~mol})$, cyclopentanone $(0.05 \mathrm{~mol})$ and ammonium acetate $(0.5 \mathrm{~mol})$ in ethanol $(250 \mathrm{~mL})$ with $1 \mathrm{~mL}$ of $30 \%$ hydrogen peroxide was boiled during $1 \mathrm{hr}$ and left to stand overnight at $20^{\circ} \mathrm{C}$. The precipitate was refluxed in $50 \mathrm{~mL}$ acetone, and then filtered to get product, which is dried in vacuum at $80^{\circ} \mathrm{C}$ for $24 \mathrm{~h}$. Melting point:241-243 ${ }^{\circ} \mathrm{C},{ }^{1} \mathrm{H}$ NMR $\left(\mathrm{CDCl}_{3}, 400\right.$ $\mathrm{MHz}): \delta=7.60(2 \mathrm{H},=\mathrm{CH}), 6.6-7.4(9 \mathrm{H}$, aromatic $)$, 3.65-3.85 $\left(18 \mathrm{H},-\mathrm{OCH}_{3}\right), 2.65-3.0\left(8 \mathrm{H},-\mathrm{CH}_{2}\right)$.

\subsection{Measurements}

${ }^{1} \mathrm{H}$ NMR spectra were recorded on a Mercury Plus $400 \mathrm{~Hz}$ spectrometer with $\mathrm{CDCl}_{3}$ as solvent. FT-IR spectra were recorded on a Perkin-Elmer Paragon1000 FTIR spectrometer. The samples were prepared as $\mathrm{KBr}$ disc. Elemental analysis was conducted on an Elementar Varioel apparatus. Mass spectra were recorded on an HP5989A mass spectrometer. UV-vis spectra were recorded by Perkin-Elmer Lambda $20 \quad$ UV-vis spectrophotometer. Fluorescence spectra were recorded by Perkin-Elmer LS50B luminescence spectrophotometer.

\subsection{Evaluation of photosentivity in DFR}

The photosensitivity of HABI photoinitiator comprised of BCIM, LCV and methoxy DSPs as photosensitizer was evaluated in the dry film. Photopolymer solutions containing a poly (styreneco-methylmethacrylate-co-methacrylic acid) as a base polymer (50wt parts), an EO-modified bisphenol-A dimethacrylate oligomer as a crosslinker (50wt parts), photoinitiators, and solvents were prepared. The solutions were coated on $16 \mathrm{um}$ thick-polyethylene terephthalate film 
using bar coater. By drying in an oven at $110^{\circ} \mathrm{C}$ for $15 \mathrm{~min}$, 30um thick-photopolymerizable films were prepared. The photoinitiator system containing BCIM, LCV and $\mathrm{C} 1$ as photosensitizer was used as reference (Scheme 3). Light source is high-pressure Hg Lamp.

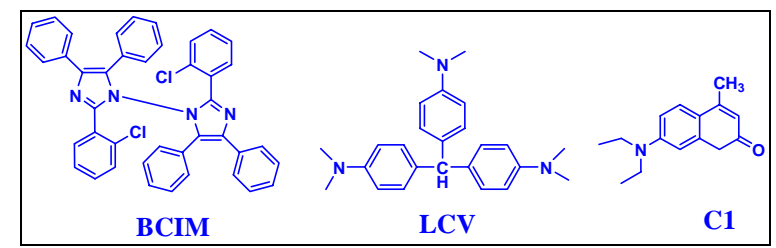

Scheme 3 Photoinitiator system as reference

\subsection{Fluorscence decay analyses}

$\mathrm{GaN}$ laser diode pumped single-photon counting fluorescence decay analyses were conducted using equipment which was described before [10-12].

\section{Results and Discussion}

3.1 Synthesis and UV absorption spectra of methoxy DSPs

To study the effect of methoxy substituent on photosensitivity of DSP, eleven methoxy DSPs were synthesized according to Scheme 3. These methoxy DSPs can be divided into three groups: momo-methoxy, di-methoxy and tri-methoxy DSP. Their UV-vis absorption spectra in chloroform are shown in Figure 1. From the Figure 1, the position and number of methoxy substituent have obvious influence on UV-absorption of DSP. The UV absorption maxima of most methoxy DSPs are about $380-400 \mathrm{~nm}$ with $\varepsilon$ of above $2 * 10^{4}$. Except for 245TMOP-DSP, the cut-off of UV-absorption spectra for all DSPs is below $450 \mathrm{~nm}$, which is expected for practical stability under the yellow light. These UV absorption character make most methoxy DSPs promising as photosensitizer for HABI.

\subsection{Photosensitivity of methoxy DSPs}

BCIM was chosen as for evaluation. To evaluate the photosensitivity of methoxy DSPs and $\mathrm{C} 1$ was chosen as a reference photosensitizer. Using a model formulation we prepared test dryfilm samples. Photosensitivity results of methoxy DSPs are shown in Figure 2. Figure 2 is showing 24DMOP-DSP was the most efficient photosensitzer among all methoxy DSPs. Also comparing with photosensitivity of $35 \mathrm{~mJ} . \mathrm{cm}^{-2}$ of $\mathrm{C} 1$ reference, the photosensitivity of 24DMOP-DSP was $14 \mathrm{~mJ} . \mathrm{cm}^{-2}$, and was increased by 1.5 times. Figure 3 is showing SEM of micro-pattern obtained test film resist which used 24DMOP-DSP as a photosensitizer, indicating excellent pattern shape with high resolution of 10 $\mu \mathrm{m}$ with $25 \mu \mathrm{m}$ resist thickness.
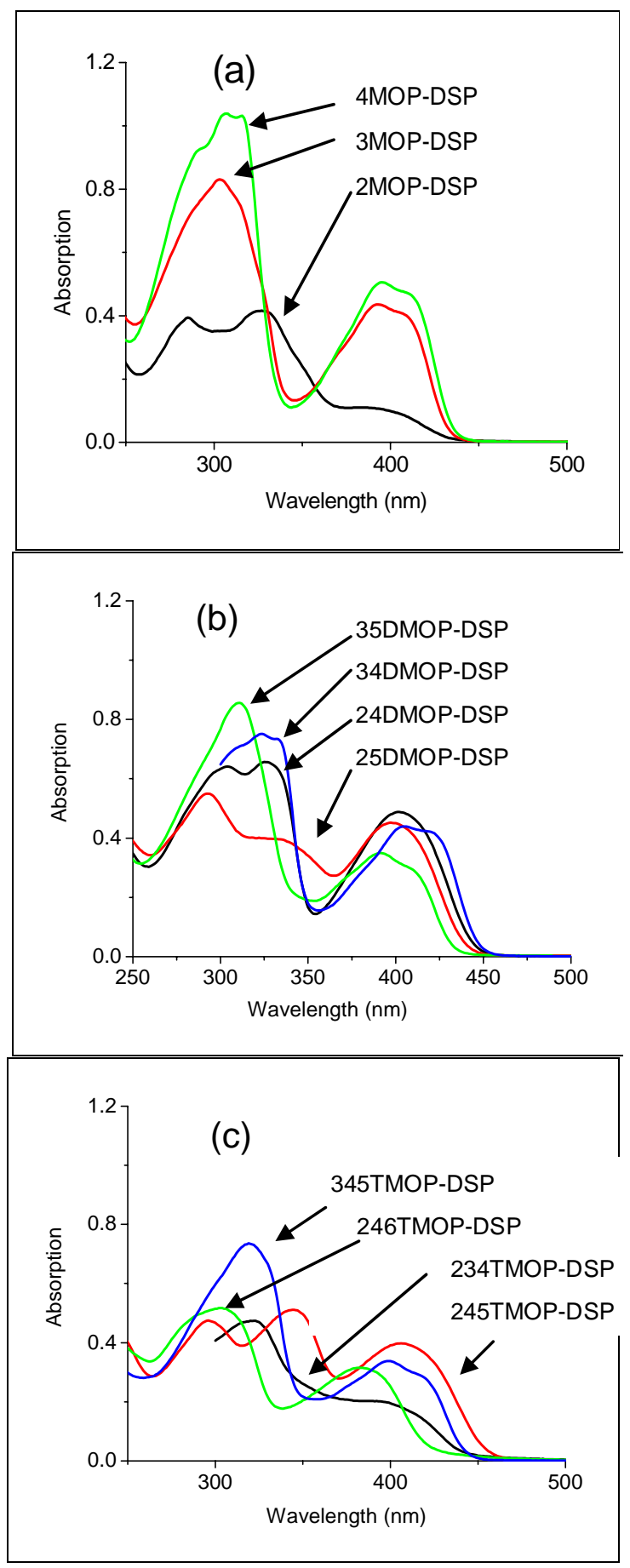

Figure $1 \mathrm{UV}$-Vis absorption spectra of all methoxyl DSPs in chloroform solution. the concentration is $2 \times$ $10^{-4} \mathrm{molL}^{-1}$ 


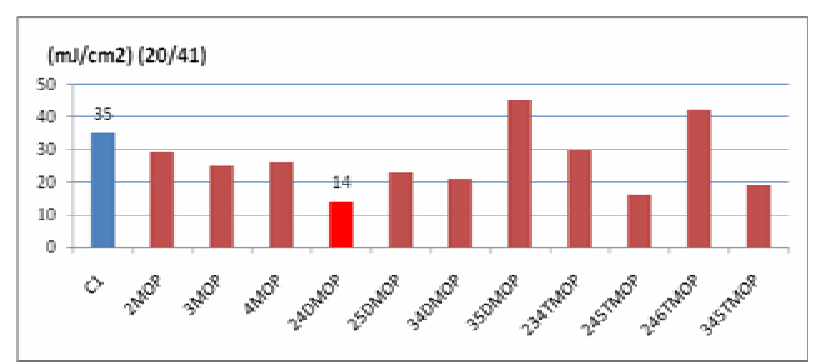

Figure 2 Photosensivity for all methoxy DSP as photosensitizer.

$\mathrm{BCIM}$ as $\mathrm{HABI}, \mathrm{LCV}$ as hydrogen donor, $\mathrm{C} 1$ as reference, high pressure $\mathrm{Hg}$ lamp as light source.

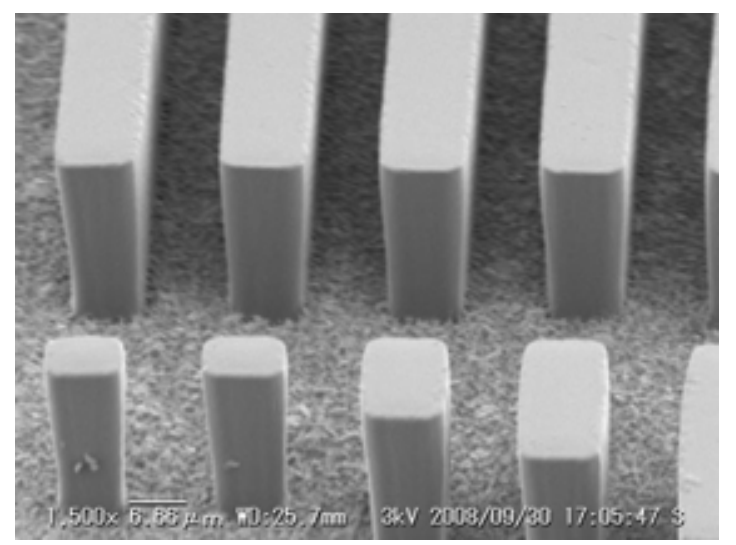

Figure 3 SEM of micro-pattern fabricated thick photoresist containing 24DMOP-DSP as photosensitizer.

High pressure Hg lamp as light source

\subsection{Mechanism study}

It was described that methoxy DSPs, especially for 24DMOP-DSP, can be used as an efficient photosentizer for HABI photoinitiator systems. We tried to know why 24DMOP-DSP is efficient. Figure 4 is showing photobleaching of 24DMOP-DSP. For instance, the absorption around $400 \mathrm{~nm}$ decreased very fast with the irradiation of $365 \mathrm{~nm}$ light. Photobleaching speed of all methoxy DSPs is shown in the Figure 5, indicating that methoxy substituent influenced on photobleaching speed of methoxy DSPs. The conversion can be calculated according to the following equation, and photobleaching speed is defined as irradiation time to $20 \%$ conversion.

$$
C=\frac{100\left(O D_{0}-O D_{t}\right)}{O D_{0}} \%
$$

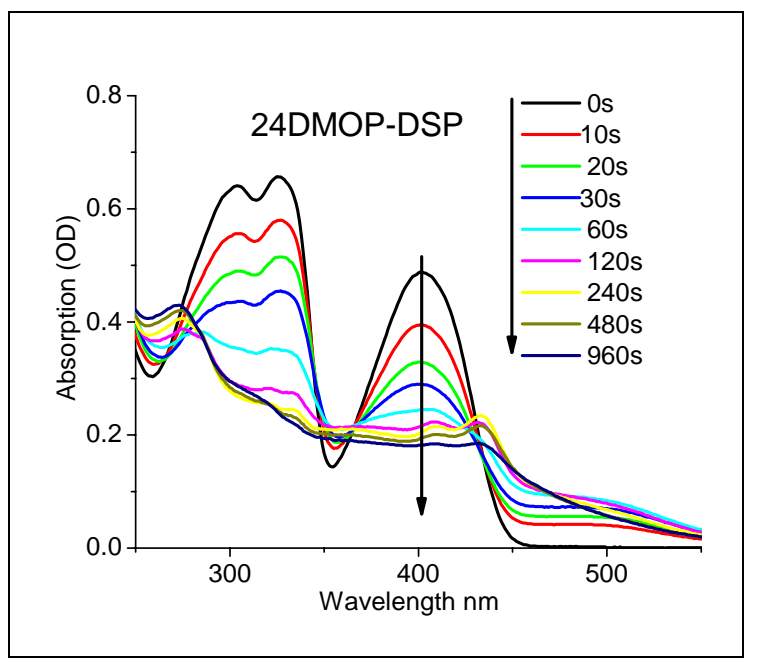

Figure 4 UV-vis absorption spectra of 24DMOP-DSP changes with the time of $365 \mathrm{~nm}$ light irradiation in chloroform. Concentration is $2 \times 10^{-4}$ molL ${ }^{-1}$

${ }^{1} \mathrm{H} \quad \mathrm{NMR}$ spectra of 3MOP-DSP in deuterated chloroform before and after irradiation are shown in Figure 6. It was clearly seen that the new signals (1', 2'and 3') appeared after irradiation, indicating configuration change of DSP. There are two $\mathrm{C}=\mathrm{C}$ groups in DSP's structure. DSP can undergo configuration change from $\mathrm{EE}$ to $\mathrm{EZ}$ to $\mathrm{ZZ}$ (Figure 6), resulting in change of UV absorption after irradiation.

The layer near to the bottom of DFR is difficult to be photo-cured due to "filter-effect" of photoinitiator systems. In the HABI photoinitiator system, the main function of photosensitizer is to absorb UV-light and to transfer its energy to HABI. Compared with conventional photosensitizer $\mathrm{C} 1$, therefore, more UV light will reach the deep layer due to photobleaching of 24DMOP-DSP as photosensitizer (shown in Figure 7), resulting in higher photosensitivity. There remain further discussion and study. This isomerization reaction may also be considered as an energy wasting side-reaction for photosensitization of BCIM by excited DSP. 

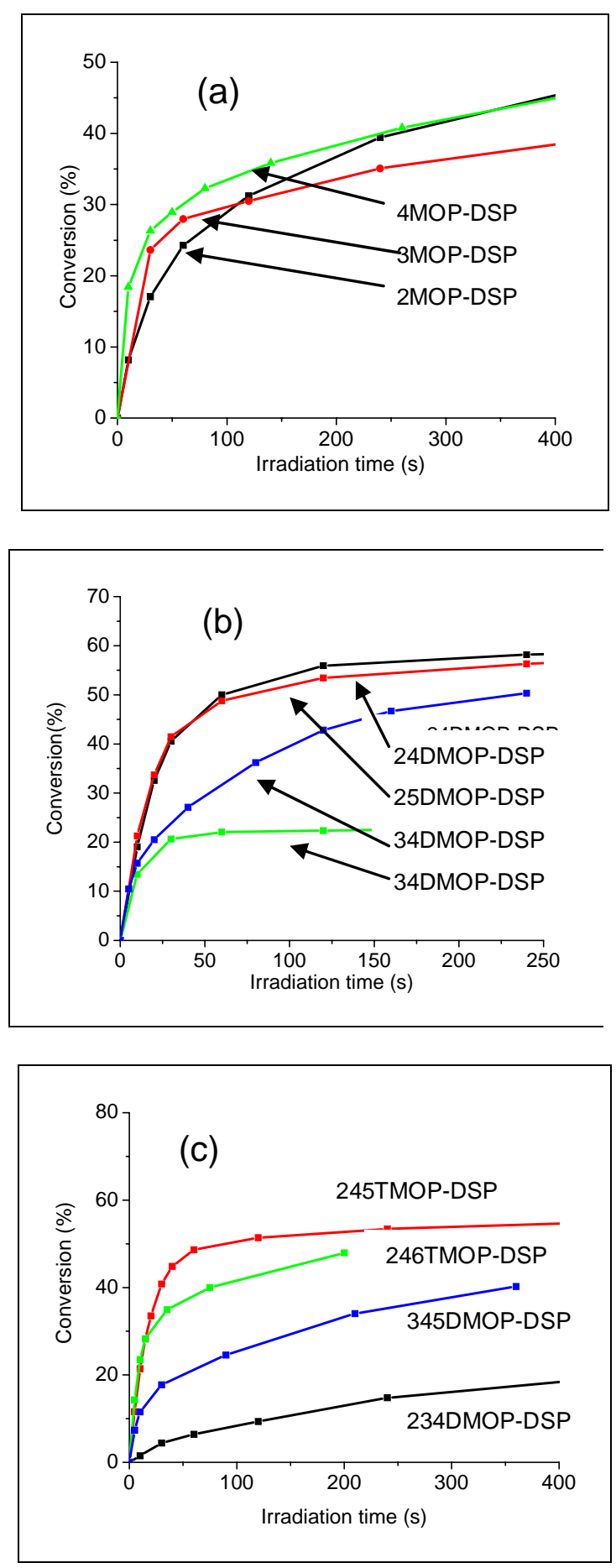

Figure 5 Photobleaching of methoxy DSPs: Conversion vs time of irradiation.

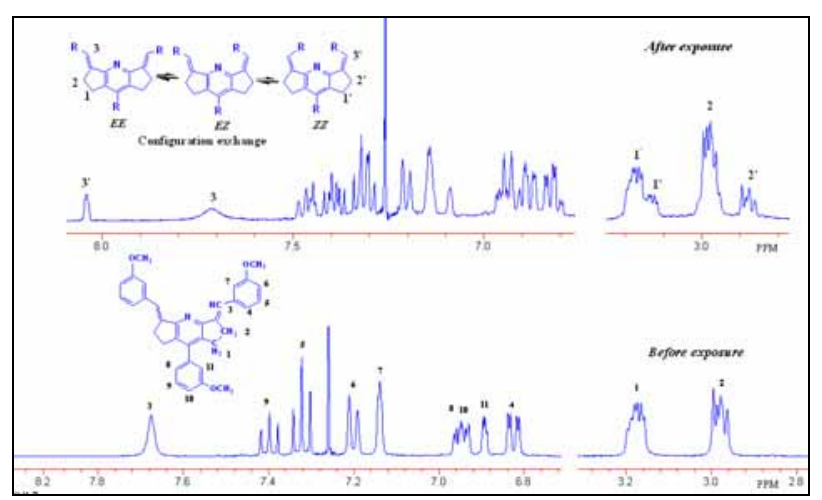

Figure $6 \mathrm{H}$ NMR spectra of $3 \mathrm{MOP}-\mathrm{DSP}$ in $\mathrm{CDCl}_{3}$ before and after irradiation of $365 \mathrm{~nm}$ light

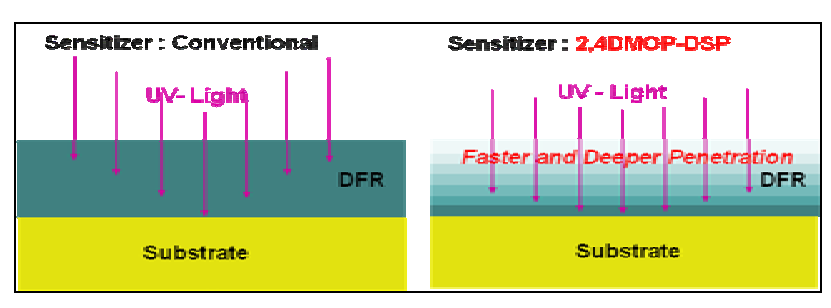

Figure 7 Proposed mechanism for effect of photobleaching property of photosensitizer for HABI systems

Two DSPs; 24DOM-DSP and 4MOP-DSP were subjected to fluorescence decay analyses in order to confirm excited singlet state energy or electron transfer path for photosensitization of DSP to HABI. This time also we asked this experiment to Tsukuba University. Equipments and procedures were shown in previous report [10]. We defined the dynamic fluorescence quenching efficiency, $\eta \mathrm{D}$, as showing in Figure 8, then obtained them from fluorescence lifetime data with and without BCIM using a single photon counting method. Results were summarized in Table 1. 24DOMP-DSP and 4MOP-DSP have medium fluorescence lifetime (3.33 and $3.46 \mathrm{~ns}$, respectively) among studied photosensitizers. The dynamic fluorescence quenching efficiency, $\eta \mathrm{D}$, for both were somewhat different $(0.40$ and 0.11 , respectively), consisting with faster photospeed of 24DMOP-DSP than 4MOP-DSP. Also among all photosensitizers studied, 24DMOP-DSP reached to maximum quenching ability and this consists with DSP`s high photospeed. 
Table 1 Fluorescence decay and quenching results

\begin{tabular}{|c|c|c|c|c|c|}
\hline $\begin{array}{c}\text { Photosensitizer } \\
\text { Observed Wavelength }\end{array}$ & ave. $/$ ns & $\eta \mathrm{D}$ & $\begin{array}{c}\text { Excitation } \\
/ \mathrm{nm}\end{array}$ & $\begin{array}{c}\text { Emission } \\
\text { observaed } \\
/ \mathrm{nm}\end{array}$ & $\begin{array}{c}\text { Photospeed } \\
\mathrm{mJ} . c m-2\end{array}$ \\
\hline DMOP-DSP & 3.33 & 0.4 & 410 & 460 & 197 \\
\hline MOP-DSP & 3.46 & 0.11 & 410 & 460 & 278 \\
\hline DBA & 11.44 & 0.28 & 410 & 460 & 175 \\
\hline C1 & 4.01 & 0.21 & 410 & 460 & 150 \\
\hline Pyraz & 2.53 & 0.08 & 410 & 450 & 240 \\
\cline { 2 - 6 } NKX-1342 & 0.6 & 0 & 410 & 480 & 572 \\
\hline N-Me-90AD & 11.66 & 0.51 & 410 & 460 & 207 \\
\hline NJ2 & 2.34 & 0.18 & 410 & 460 & 191 \\
\cline { 2 - 6 } & 1.79 & 0.09 & 410 & 460 & 179 \\
\hline NJ1 & 10.97 & 0.26 & 410 & 460 & 174 \\
\hline ACR-2 & & & & &
\end{tabular}

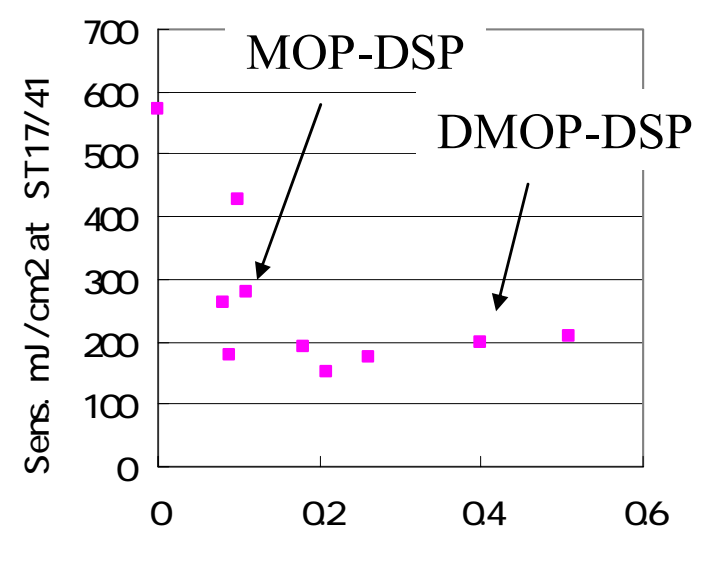

Figure 8 Fluorescence quenching efficiency and photosensitivity

Fluorescence quenching efficiency ( $n \mathrm{D})$ :

Fluorescence quenching efficiency efficiency was derived from fluorescent lifetime measurement using eq. (1)

$$
\eta \mathrm{D}=1-\mathrm{T} / \mathrm{T}
$$

$\mathrm{T}_{0}$ : fluorescence lifetime without quencher [ns]

$T$ : fluorescence lifetime with quencher [ns]

Quencher: BCIM, 1.5 wt parts

\section{Conclusion}

Eleven methoxy DSPs were newly synthesized and evaluated as a photosensitizer for
HABI photoinitiator system in DFR. Among all methoxy DSPs, 24DMOP-DSP was the most photosensitive. Compared with $35 \mathrm{~mJ} . \mathrm{cm}^{-2}$ of $\mathrm{C} 1$ as reference, the photosensitivity for 24DMOP-DSP as photosensitizer is about 14 $\mathrm{mJ} . \mathrm{cm}^{-2}$, and is increased about $150 \%$. The photobleaching property of 24DMOP-DSP, derived from configuration change of $E$ to $Z$, is one of key factors to high performance of 24DMOP-DSP such as high photosensitivity and excellent pattern. DSP undergoes a photoisomerization around its double bond and its excited singlet state is easily quenched by BCIM.

\section{Acknowledgement}

We express many thanks to professor Arai and professor Nishimura of Tsukuba University for measuring time-resolved fluorescence decay.

\section{References}

1. J. P. Fouassier, "Photoinitiation, Photopolymerization, and Photocuring Fundamentals and Applications", Hanser, New York 1995.

2. C. Roffy, "Photogeneration of Reactive Species for UV-Curing”, Wiley, New York 1997.

3. C. Decker, Prog. Polym. Sci., 1996, 21, 593.

4. B. M. Monroe,G. C. Weed, Chem. Rev., 1993, 93, 435

5. M.R. Mckeever, W.O. Patent, 99/46644 (1999).

6. $\quad$ L.A. Cescon, U.S. Patent, 3,784,557 (1974).

7. T.M. Sheets, U.S. Patent, 4,622,286 (1986).

8. X.Z. Qin, A. Liu, A.D. Trifunac, and V.V. Krongauz, J. Phys.Chem., 1991, 95, 5822.

9. V. G. Pivovarenko, A. V. Grygorovych, V. F. Valuk, A. O.Doroshenko, Journal of Fluorescence, 2003, 13, 479.

10. Y. Nishimura, M. Kamada, M. Ikegami, R. Nagahata, T. Arai, J. Photochem. Photobiol. A:Chem., 178(2006)150.

11. M.Kaji, Y.Muramatsu, Y.Nishimura, T.Arai, $J$. Photopolym. Sci. Technol., 20 (2), (2007)265 